\title{
LOS ESPECTADORES Y ESPECTADORAS DE LA CIBERVIOLENCIA DE GÉNERO
}

\author{
VIEWERS OF GENDER CYBERVIOLENCE
}

\author{
Trinidad Donoso Vázquez \\ Facultad de Educación de la Universidad de Barcelona \\ trinydonoso@ub.edu
}

María José Rubio Hurtado

Facultad de Educación de la Universidad de Barcelona

mjrubio@ub.edu

Ruth Vilà Baños

Facultad de Educación de la Universidad de Barcelona

Ruth_vila@ub.edu

\section{RESUMEN}

La finalidad del estudio fue analizar el papel que tienen las y los jóvenes cuando son observadores de ciberviolencias de género, identificando qué características de ellos/as pueden aumentar la probabilidad de actuar con complicidad con el ciberagresor, y cuáles de actuar con ayuda a la víctima. Se aplicó una encuesta mediante cuestionario on-line a una muestra de 4536 adolescentes de $3^{\circ}$ y $4^{\circ}$ de Enseñanza Secundaria Obligatoria procedentes de las comunidades de Cataluña, Aragón, Galicia, Andalucía, Islas Baleares y Canarias. El análisis, mediante la técnica de los árboles de decisión, reveló que las variables que tienen una mayor probabilidad de influir en las actuaciones de los observadores/as de ciberviolencia son el sexo, tener características de vulnerabilidad, la edad, haber sufrido acoso y la percepción de los riesgos de las redes sociales en internet. Las chicas tienen mayor probabilidad de actuar como defensoras de la víctima, probabilidad que aumenta cuando sienten que tienen alguna característica que las hace vulnerables ante las ciberviolencias. A más edad, mayor probabilidad de tener el perfil de complicidad con el agresor, probabilidad que aumenta siendo chicos que se sienten vulnerables, que han sufrido ciberacoso en alguna ocasión, y que perciben los riesgos que tienen las redes sociales en internet. Consecuentemente, son los que tienen menor probabilidad de actuar defendiendo a la víctima. Los resultados obtenidos alientan la necesidad de emprender acciones educativas que ayuden a paliar el fenómeno de la ciberviolencia de género, enfatizándose la idea de que no deben estar centradas en los dos polos del fenómeno, agresor y víctima, sino incluir también a las personas observadoras. Las acciones también deberían orientarse a la formación del profesorado, pues los docentes son un elemento 
principal para llevar a cabo cambios en el entorno educativo, entorno en el que comienza y se expande la ciberviolencia de género.

Palabras clave: ciberviolencia de género, adolescentes, conductas observadores, acciones educativas

\begin{abstract}
The purpose of the study was to analyze the role of young people when they are observers of gender cyberviolence, identifying what characteristics of them may increase the likelihood of complicity with cyberagresor, and which ones to act with assistance to the victim. An online questionnaire survey was applied to a sample of 4536 adolescents from third and fourth courses of Compulsory Secondary Education from Spain (Catalonia, Aragón, Galicia, Andalucía, Islas Baleares and Islas Canarias). The analysis, using the decision trees technique, revealed that the variables that have a greater probability of influencing the actions of the cyberviolence observers are the sex, vulnerability characteristics, age, harassment and perception of the risks of social networks on the internet. Girls are more likely to act as defenders of the victim, a likelihood that increases when they feel they have some feature that makes them vulnerable to cyber-violence. Older, they are more likely to have the complicity profile with the aggressor, a likelihood that increases when the boys feel vulnerable, have experienced cyberbullying on some occasion, and perceive the risks that social networks have on the internet. Consequently, they are the ones that are less likely to act defending the victim. The results obtained encourage the need to undertake educational actions that help to alleviate the phenomenon of gender cyber violence, emphasizing the idea that they should not be focused on the two poles of the phenomenon, aggressor and victim, but also include observers. Actions should also be geared towards teacher training, as teachers are a key element in bringing about changes in the educational environment, where cyberviolence begins and expands.
\end{abstract}

Key words: gender cyber violence, adolescents, observer behavior, educational actions.

\title{
1. INTRODUCCIÓN
}

Internet es el lugar de residencia de los jóvenes (White y Le Cornu, 2011). No un lugar de visita, o de paso o de tránsito. Internet es el lugar donde los jóvenes expresan sus afectos y los reciben, donde aprenden, donde desarrollan identidades, en definitiva, el lugar donde se reafirman y viven. El Instituto Nacional de Estadística (2015) cifra en más del 85\% el uso de Internet por los jóvenes alcanzando el 99\% cuando sobrepasan los 15 años. En la última encuesta (2016) entre la población de 10 a 15 años la tasa de uso de internet es prácticamente universal (94,9\%). Por primera vez el número de menores usuarios de Internet supera al de usuarios del ordenador. Por sexo, las diferencias de uso de ordenador y de Internet apenas son significativas.

No cabe duda de que la comunicación y la interacción humana han recibido un espaldarazo y desarrollo gracias a las tecnologías de la información y la comunicación (TIC). Pero estas mismas tecnologías sirven para crear peligros a los que adolescentes y jóvenes están especialmente expuestos por su momento evolutivo y por lo que significa para ellos y ellas internet: su lugar de residencia.

El ciberacoso o ciberviolencia se puede definir como una forma de intimidación, acoso y malos tratos por parte de un individuo o grupo hacia otro, implicando el uso de medios tecnológicos como canal de agresión (Ortega, Calmaestra y Mora, 2008; Smith, 2006). Entre las conductas que puede realizar la persona agresora se encuentran el envío y difusión de mensajes ofensivos, 
amenazas, la difusión de rumores sobre la víctima, la violación de intimidad, la exclusión social, o la suplantación de la identidad (Calmaestra, 2011).

Internet posibilita nuevas posibilidades y facilidades para el acoso:

a. La facilidad de acceso y consumo de estos espacios virtuales (Finn y Atkinson, 2009; Hand, Chung, y Peters, 2009).

b. El anonimato que se posibilita en las interacciones en ciertos espacios virtuales. (Hand et al., 2009). La dificultad de rastreo de la autoría de ciertas acciones llevadas a cabo mediante la red puede acrecentar la sensación de impunidad para el agresor.

c. La gran diversificación de las formas de acoso que la red permite (Finn y Atkinson, 2009). Utilizando las redes sociales, los espacios virtuales, las formas de comunicación como WhatsApp o los emails, se ha ampliado el rango de posibilidades a través de las cuales ejercer un acoso.

d. Esta diversificación de las formas de acoso a través de la red, generan una contundencia y posibilitan una insistencia desconocida hasta la fecha. El agresor puede destinar las 24 horas del día a acosar a su víctima y hacerlo desde múltiples dispositivos o espacios de forma simultánea.

e. Otro aspecto del ciberacoso es que posibilita acosar a pesar de mantener una distancia geográfica. No importa dónde se encuentren acosador y víctima, pues el acoso puede darse con la misma o incluso mayor insistencia que cuando el acoso se daba de forma directa y personal.

f. El agresor puede tomar el control de las esferas pública y privada de la víctima (Hand, et al., 2009). El objetivo de cualquier acosador será limitar el acceso de la víctima al mundo exterior (Finn y Atkinson, 2009), y a la vez, controlar todo lo que la víctima realice en su vida privada.

g. Finalmente, es interesante señalar que el acoso a través de las redes sociales o espacios virtuales agrega una sensación de falta de control importante para la víctima. Esté donde esté, a la hora que sea y a través de cualquier medio o dispositivo tecnológico, la persona puede estar siendo acosada o controlada. Estos hechos aumentan la sensación de angustia por parte de la víctima, que le otorga más poder al agresor.

La repercusión que está teniendo el ciberacoso en jóvenes es considerable dada su amplitud y las consecuencias que conlleva: malestar psicológico, baja autoestima, depresión, ansiedad, síntomas psicosomáticos, llegando hasta el suicidio. (Arsenault, Bowes y Shakoor, 2010; Menesini, Nocentini y Palladino, 2009; Ttofi, Farrington, Lösel y Loeber, 2011; Veenstra, Siegwart, Munniksma y Dijkstra, 2010). También tiene un impacto negativo en los espectadores no involucrados en problemas de intimidación (Gini, Pozzoli, Borghi y Franzoni, 2008).

Los datos de la ciberagresión en jóvenes son muy variables. Según la revisión de estudios realizada por Calvete, Orue, Estévez, Villardón, y Padilla, (2010) la prevalencia del acoso online 
va de $1,7 \%$ a $35,7 \%$. Solo la prevalencia del sexting en adolescentes oscila entre el 7\% y el $27 \%$ (Cooper, Quayle, Jonsson y Svedin, 2016).

Aunque no son concluyentes las investigaciones sobre la prevalencia de uno u otro sexo como la figura agresora, hay que recalcar que las diferencias entre chicos y chicas van más allá de las cifras:

Las chicas describen en sus perfiles las situaciones más personales e íntimas (...) se exponen más que los chicos, reciben más solicitudes de amistad, más propuestas de desconocidos y proposiciones sexuales, conocen competiciones donde son puntuadas por su físico. En definitiva, las chicas viven en las redes un riesgo a ser acosadas y una vulnerabilidad mayor que los chicos. A esta exposición se suman las diferencias ante situaciones de violencia: los chicos minimizan las agresiones ejercidas, las chicas minimizan las recibidas (Estébanez y Vázquez, 2013: 102).

Un caso especial son las violencias que se ejercen en internet en función del género. Las violencias de género 2.0 son violencias que se ejercen sobre mujeres, por la posición que se les ha asignado en la construcción histórica de la categoría hombre-mujer, pero también sobre aquellos y aquellas que trasgreden el orden social generizado. Este orden social imperante se traspasa a los espacios virtuales atribuyendo lugares determinados, específicos, constreñidos y subalternos a las mujeres y a ciertas categorías de personas que son discriminadas por transgredir las formas de conductas obligatorias que propone el imaginario social (Donoso, Vilà, Rubio y Prado, 2016).

La imagen que muestra la adolescencia en las redes sociales no está exenta de estereotipos de género: mientras que las chicas utilizan estrategias para parecer más atractivas, los chicos lo hacen para parecer más "machos", reforzando así la reproducción de un modelo de feminidad y masculinidad muy anclado en patrones sexistas, en modelos hegemónicos. (Méndez, Villar y Barreiro, 2015: 172).

Un corpus empírico que está apareciendo con fuerza en este tema es el que ahonda, no en las víctimas o las personas agresoras, sino en las personas espectadoras (Salmivalli, Kärnä, y Poskiparta, 2011; Salmivalli y Poskiparta, 2012; Menesini, Nocentini y Palladino, 2012). Para reducir las ciberagresiones, no necesariamente tenemos que cambiar a las víctimas y hacerlas "menos vulnerables". Por otra parte, el comportamiento de los agresores es difícil cambiarlo si no alteramos otros elementos del contexto. Con frecuencia los agresores reciben una retroalimentación positiva de sus acciones o recompensas, del tipo prestigio social o estatus entre los pares. El mensaje de estos estudios es que el comportamiento de los espectadores importa. Las víctimas que sienten que los compañeros las apoyan y defienden se sienten mejor que las víctimas sin defensores. Las víctimas defendidas están menos deprimidas y ansiosas, tienen una mayor autoestima, y son menos rechazadas por sus iguales que las víctimas sin defensores. Además, se ha demostrado que en las aulas donde reforzar al agresor es un comportamiento normativo (es decir, típico de muchos niños), la probabilidad de intimidar a otros es superior.

Esto no significa que los agresores individuales y las víctimas no necesiten atención. Los investigadores en este campo comparten la opinión de que los espectadores son una clave para las 
intervenciones eficaces y sostenibles, al mismo tiempo que una intervención individualizada cuando se presenten casos agudos.

El estudio de Salmivalli, Kärnä y Poskiparta (2011), identificó seis roles diferentes que los y las participantes pueden tener en situaciones de ciberacoso. Una persona puede asumir el rol de víctima, de agresora, de ayudante del agresor, de reforzadora del agresor, de forastero o defensora de la víctima. Dada la escasez de estudios sobre el comportamiento de los y las observadores en la población española, las aportaciones que se hagan en este ámbito, contribuirán a aportar estrategias educativas para erradicar la violencia.

La finalidad de nuestro estudio está en esta línea, y persigue analizar el papel que tienen las y los jóvenes cuando son observadores de ciberviolencias de género, identificando qué características de los jóvenes pueden aumentar la probabilidad de actuar con complicidad con el ciberagresor, y cuáles con ayuda a la víctima.

\section{MÉTODO}

Se ha realizado un estudio por encuesta usando como instrumento de recogida de datos el Cuestionario de ciberviolencias de género (Donoso, 2014), al cual respondieron de forma online durante el periodo marzo-junio de 2015, adolescentes de Educación Secundaria Obligatoria de las comunidades de Cataluña, Aragón, Galicia, Andalucía, Islas Baleares y Canarias. Las dimensiones del Cuestionario de ciberviolencias de género (tabla 1) se relacionan con los siguientes aspectos teóricos: Competencias y uso de entornos digitales, percepción de internet como entorno violento, percepción de impunidad de la red, percepción de tener características personales de vulnerabilidad, y respuestas y formas de actuar cuando se observan conductas de violencia de género 2.0. En nuestro estudio seguimos la clasificación para los roles de los espectadores y espectadoras del ciberacoso basado en Salmivalli, Kärnä y Poskiparta (2011). Concretamente, los asistentes (assistant) son chicos y chicas que se unen de una forma activa a los agresores, los reforzadores (reinforcer) proporcionan retroalimentación positiva a los agresores (por ejemplo, riendo o animando), los pasivos (outsider) se retiran de las situaciones de acoso y los defensores (defender) toman partido por las víctimas, reconfortándolas y apoyándolas. A nivel de análisis, dada la tipología de respuestas obtenidas, estos cuatro perfiles se han dicotomizado artificialmente en dos: los y las defensoras hacen referencia al perfil defensor de la víctima; y los tres perfiles restantes (reforzadores, pasivos y asistentes) se han agrupado bajo el perfil de complicidad con el ciberagresor. 
TRINIDAD DONOSO VÁZQUEZ, Ma JOSÉ RUBIO HURTADO Y OTROS: Los espectadores...

\begin{tabular}{|c|c|c|}
\hline \multicolumn{3}{|c|}{ Dimensiones de análisis } \\
\hline \multicolumn{3}{|c|}{ Datos sociodemográficos: curso, sexo, pareja, sexualidad. } \\
\hline \multicolumn{3}{|c|}{ Percepción de internet como entorno violento } \\
\hline \multicolumn{3}{|c|}{ ¿Te han acosado alguna vez por Internet o móvil? Dicotómica } \\
\hline \multicolumn{3}{|c|}{ ¿Las personas son más violentas en los entornos online que en el cara a cara? Dicotómica } \\
\hline \multicolumn{3}{|c|}{ Escala de Riesgos en internet. 5 ítems Likert } \\
\hline \multicolumn{3}{|c|}{$\begin{array}{l}\text { Percepción de impunidad de la red } \\
\text { Cuando alguien hace alguna cosa negativa o ilegal en entornos online, ¿crees que generalmente se le castiga? } \\
\text { Dicotómica }\end{array}$} \\
\hline \multicolumn{3}{|c|}{$\begin{array}{l}\text { Percepción de tener características personales de vulnerabilidad } \\
\text { ¿Crees que posees alguna característica por la que se podrían meter contigo? Dicotómica }\end{array}$} \\
\hline \multirow[t]{2}{*}{$\begin{array}{c}\text { Respuestas como } \\
\text { observador/a }\end{array}$} & $\begin{array}{l}\text { Perfil defensor de la } \\
\text { víctima }\end{array}$ & Defensores: Han defendido o ayudado a la persona agredida \\
\hline & $\begin{array}{l}\text { Perfil de complicidad con } \\
\text { el ciberagresor }\end{array}$ & $\begin{array}{l}\text { Reforzadores: Han animado al que lo está haciendo } \\
\text { Pasivos: No han hecho nada } \\
\text { Asistentes: Han ayudado al que lo está haciendo }\end{array}$ \\
\hline
\end{tabular}

Tabla 1. Dimensiones del Cuestionario. Elaboración propia

La selección de la muestra fue mediante muestreo aleatorio por conglomerados, siendo los conglomerados los centros de secundaria de cada región participante en el estudio. A los centros se accedió mediante contacto personal con el equipo directivo. Del total de estudiantes incluidos en la muestra, contestaron finalmente 4536 adolescentes, lo que supone un margen de error de \pm 0.014 , para un nivel de confianza del $95,5 \%$ en poblaciones infinitas, donde p y q son iguales.

Se ha realizado un análisis descriptivo de todas las variables del estudio, en relación con las medidas de tendencia central y desviación en el caso de las variables cuantitativas y frecuencias de aparición de las categorías para las variables cualitativas.

También se ha aplicado la técnica de Minería de datos árboles de decisión CHAID, que prepara, sondea y explora los datos para obtener la información oculta en ellos abordándose la solución a problemas de predicción, clasificación y segmentación (Pérez López y Santín, 2007). Los Árboles de decisión crean un modelo de clasificación basado en diagramas de flujo con forma arborescente, y clasifican casos en grupos (Román y Lévy, 2003). Este análisis exploratorio nos lleva a identificar variables importantes y sus interacciones enfocadas a la segmentación (Pérez López, 2011). En la investigación se trabajó con una muestra elevada (4536 adolescentes), cumpliendo con la recomendación de aplicación de árboles de decisión de muestras no inferiores a 1000 casos (Berlanga, Rubio y Vilà, 2013). Los árboles de decisión pueden ser útiles por tanto para identificar los elementos clave para que los jóvenes cuando observan ciberviolencias de género actúen ayudando a la víctima en vez de siendo cómplices con la violencia observada. Para ello se ha utilizado de variable dependiente: Respuestas como observador/a dicotomizando las respuestas entre: cómplices o ayudan a la víctima. Y se han añadido las siguientes variables independientes:

- Cuando alguien hace alguna cosa negativa o ilegal en entornos online, ¿crees que generalmente se le castiga? 
- ¿Te han acosado alguna vez por Internet o móvil?

- Riesgos en internet

- Curso

- Sexo

- ¿Actualmente tienes pareja?

- Características de vulnerabilidad: ¿Crees que posees alguna característica por la que se podrian meter contigo?

- Sexualidad

- ¿Las personas son más violentas en los entornos online que en el cara a cara?

\section{RESULTADOS}

Los y las adolescentes participantes están escolarizados en $3^{\circ}$ y $4^{\circ}$ de ESO. La muestra presenta porcentaje similar de chicas y chicos (52\% y $48 \%$ respectivamente). El $26 \%$ dice tener pareja afectiva y casi la mitad (48\%) cree tener alguna característica de vulnerabilidad para poder padecer violencia. Mayoritariamente la muestra se considera heterosexual (89\%), aunque hay diversidad de opciones.

Aproximadamente 2 de cada 10 adolescentes han sido acosados o acosadas en entornos virtuales. Mayoritariamente creen que hay más violencia online que offline, el $90 \%$ de las y los adolescentes así lo manifiestan. Además, el $85 \%$ piensa que no se castiga en general a las personas que actúan de forma ilegal o dañina en la red. En general, los y las adolescentes perciben riesgo en algunas conductas online, entre las que destacan "Poner información personal en la red" o "Chatear repetidas veces con una persona de la cual no tienes indicios de quién es". Pero no perciben tan peligroso colgar fotos o videos personales.

El 57\% tiene respuestas de ayuda a la víctima cuando observa ciberviolencias de género. No obstante, un $43 \%$ de los y las adolescentes actúan con complicidad con la persona agresora (tabla 2).

\begin{tabular}{|clc|}
\hline Perfil de complicidad con el ciberagresor/a: & 1950 & $43 \%$ \\
He animado al que lo está haciendo & 37 & $1,8 \%$ \\
No he hecho nada & 1110 & $56,9 \%$ \\
He ayudado al que lo está haciendo & 44 & $2,25 \%$ \\
Perfil defensor de la víctima: & 2586 & $57 \%$ \\
He defendido o ayudado a la persona agredida &
\end{tabular}

Tabla 2. Respuestas ante la observación de las violencias de género 2.0. Elaboración propia

\section{Perfil defensor de la víctima y perfil de complicidad con el ciberagresor}

La tabla resumen del modelo (tabla 3) nos informa de las variables incluidas en el modelo, en este caso de las 9 variables independientes que habíamos seleccionado, han estado incluidas 5, lo que 
significa que éstas son las más relevantes para definir cuándo las respuestas ante las ciberviolencias de género son de ayuda a la víctima o por el contrario, de complicidad. Concretamente son relevantes: sexo, poseer características de vulnerabilidad, curso escolar, haber sufrido acoso y percibir en mayor medida los riesgos de las redes sociales en internet. También podemos apreciar que el árbol ha alcanzado los 5 niveles de profundidad.

Resumen del modelo

\begin{tabular}{|c|c|c|}
\hline \multirow[t]{7}{*}{ Especificaciones } & Método de crecimiento & CHAID \\
\hline & Variable dependiente & Respuestas2 \\
\hline & Variables independientes & $\begin{array}{l}\text { 5. Cuando alguien hace alguna cosa negativa o ilegal en } \\
\text { entornos online, ¿crees que generalmente se le castiga?, } \\
\text { 7. ¿Te han acosado alguna vez por Internet o móvil?, } \\
\text { totalRiesgo, Curso, Sexo, ¿Actualmente tienes pareja?, } \\
\text { ¿Crees que posees alguna característica por la que se } \\
\text { podrían meter contigo?, sexualidad2, } 4 \text {. ¿Las personas } \\
\text { son más violentas en los entornos online que en el cara } \\
\text { a cara? }\end{array}$ \\
\hline & Validación & Ninguna \\
\hline & Máxima profundidad de árbol & 5 \\
\hline & Mínimo de casos en un nodo filial & 100 \\
\hline & Mínimo de casos en un nodo parental & 50 \\
\hline \multirow[t]{4}{*}{ Resultados } & Variables independientes incluidas & $\begin{array}{l}\text { Sexo, ¿Crees que posees alguna característica por la que } \\
\text { se podrían meter contigo?, Curso:, 7. ¿Te han acosado } \\
\text { alguna vez por Internet o móvil?, totalRiesgo }\end{array}$ \\
\hline & Número de nodos & 15 \\
\hline & Número de nodos terminales & 8 \\
\hline & Profundidad & 4 \\
\hline
\end{tabular}

Tabla 3. Tabla resumen del modelo. Elaboración propia

El diagrama del árbol (fig. 1) muestra una representación gráfica del modelo a través de nodos que representan categorías de variables. Todas las variables son tratadas como nominales y cada nodo contiene una tabla de frecuencias que muestra el número de casos (frecuencia y porcentaje) y un gráfico para cada categoría de la variable dependiente. El Nodo 0 del árbol describe la variable dependiente: porcentaje de los jóvenes cuyas respuestas son de ayuda a la víctima (57\%) y los que tienen respuestas de complicidad con el agresor (43\%). Seguidamente observamos como la variable dependiente se ramifica en dos nodos, pertenecientes a la variable sexo (femenino, masculino), indicando que ésta es la variable principal predictora. 


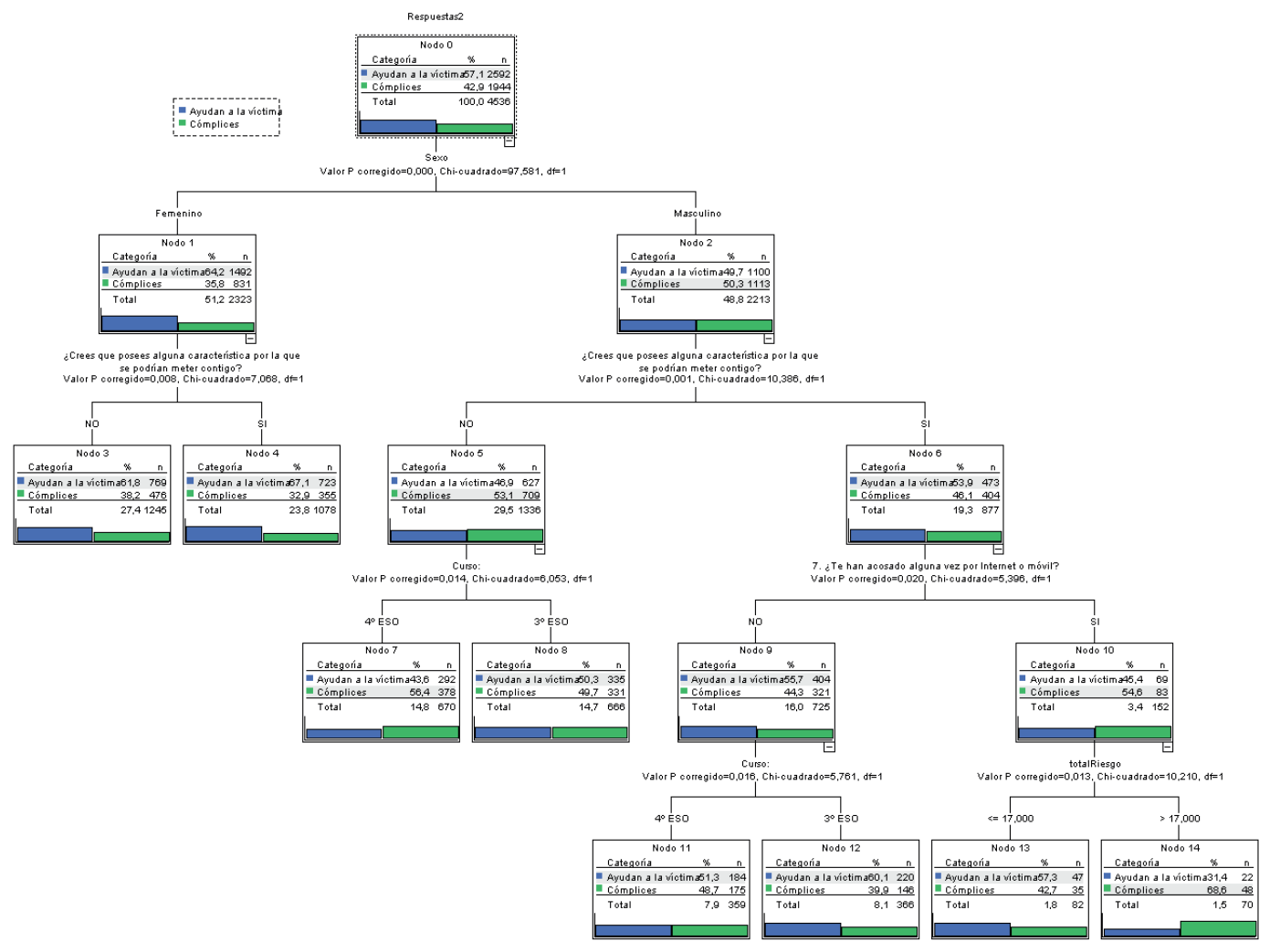

Figura 1. Representación grática del diagrama de árbol. Elaboración propia

El Nodo 1 ofrece información sobre las chicas, donde vemos que su porcentaje de respuestas de ayuda a la víctima es superior, un 64\% (ante la mitad de los chicos). Del mismo modo, los chicos actúan en mayor medida como cómplices con el agresor (en la mitad de los casos, respecto a sólo el 35\% de las chicas). El nodo de las chicas se ramifica inmediatamente en si sienten o no que tienen alguna característica de vulnerabilidad para ser cibervíctimas de violencia de género, siendo la probabilidad de tener respuestas de ayuda a la víctima mucho más alta cuando las chicas sienten que tienen alguna característica que las hace vulnerables ante las ciberviolencias.

El nodo 2, de los chicos, se vuelve a ramificar en los nodos 5 y 6 , pertenecientes a la misma variable de vulnerabilidad, mostrando que al igual que en las chicas, los que se sienten vulnerables son los que tienen más probabilidad de responder ayudando a la víctima (con un porcentaje del 54\%, respecto al $47 \%$ de los chicos que no se sienten vulnerables). Los chicos que no se sienten vulnerables tienen un 53\% de probabilidad de actuar como cómplices ante las ciberviolencias. Y si además cursan $4^{\circ}$ de la ESO, este porcentaje aumenta hasta el $56 \%$, respecto al $50 \%$ de los de $3^{\circ}$ de ESO.

En el caso de los chicos que se sienten vulnerables, el nodo 6 se vuelve a ramificar en los nodos 9 y 10, pertenecientes a la variable haber sufrido acoso. Curiosamente, los chicos que se 
sienten vulnerables y han sufrido acoso alguna vez, son los que tienen mayor probabilidad de actuar como cómplices con los agresores cuando observan ciberviolencia (55\%) respecto a aquellos que no la han sufrido (44\%). La probabilidad de actuar con complicidad con el agresor aumenta hasta el $69 \%$ cuando se trata de chicos que se sienten vulnerables, que han sufrido ciberviolencia alguna vez, y que además perciben en mayor medida los riesgos que tiene internet.

Finalmente, el nodo 9, referente a los chicos que se sienten vulnerables pero que nunca sufrieron ciberacoso, se ramifica en el último nivel entre los nodos 11 y 12 , respecto al curso. Siendo los de tercer curso los que tienen mayor probabilidad de actuar ayudando a la víctima (60\% respecto al $51 \%$ de lo de $4^{\circ}$ de ESO).

\section{DISCUSIÓNY CONCLUSIONES}

Los resultados de nuestro estudio concuerdan con los de Calvete et al. (2010), sobre la prevalencia del acoso en internet. Se sitúa en la zona inferior del rango, pero ha de anotarse que la investigación que presentamos se centra sólo en las ciberviolencias en función del género, aquellas que se dan contra mujeres y contra personas que trasgreden el orden social generizado. Seguramente si las conductas analizadas en el cuestionario fueran más extensas, el porcentaje de acoso habría sido más elevado. Aun así, que 2 de cada 10 adolescentes hayan sido acosados o acosadas en entornos virtuales es una cifra que requiere atención por parte de la sociedad y de los entornos educativos.

Otro dato que considerar y que debe hacer reflexionar a las instituciones es la percepción que tienen estos jóvenes de la mayor cantidad de violencia online que offline, $90 \%$ de las y los adolescentes así lo perciben. No podemos corroborar este dato con otras investigaciones, pues en otros estudios, como los de Martínez, Sendín y García, (2013), los jóvenes no prestan especial atención al acoso online.

La impunidad en internet es otro elemento preocupante. El 85\% de la muestra piensa que no se castiga en general a las personas que actúan de forma ilegal o dañina en la red.

La variable sexo es el mejor predictor para el tipo de respuesta ante las ciberviolencias de género. Las chicas son las que tienen mayor probabilidad de actuar como defensoras de la víctima. Otras variables incluidas en el modelo son: tener características de vulnerabilidad, curso, haber sufrido acoso y percepción de los riesgos de las redes sociales en internet.

A más edad, mayor probabilidad de tener el perfil de complicidad con el agresor. Aunque los estudios sobre las personas que son espectadoras de la violencia no son muy corrientes, es cierto que las investigaciones apuntan a que las ciberagresiones aumentan con la edad (Garmendia, Garitaonandia, Martínez y Casado, 2011).

La probabilidad más alta de actuar ayudando a la víctima (67\%) se da en las chicas que sienten que tienen alguna característica que las hace vulnerables ante las ciberviolencias. Este colectivo además es el que tiene consecuentemente, la probabilidad más baja de actuar con complicidad con el agresor (33\%).

La probabilidad más alta de actuar con complicidad con el agresor (69\%) se da en los chicos que se sienten vulnerables, que han sufrido ciberacoso en alguna ocasión, y que perciben los riesgos que tienen las redes sociales en internet. Consecuentemente, son los que tienen menor probabilidad 
de actuar defendiendo a la víctima (31\%). El impacto de la ciberviolencia se va perpetuando en un bucle. Quien la ha sufrido se convierte a su vez, si no en agresor, sí en cómplice del agresor.

Tomamos la opción de integrar al perfil neutro dentro del perfil de complicidad. Consideramos que el "no hacer nada" o situarse en una posición de outsider cuando se está realizando un acoso debe considerarse una actitud pro-refuerzo del acoso en sí. De cara a las campañas de prevención o en las intervenciones educativas, utilizar una estrategia de responsabilidad hacia los perfiles neutros tendrá repercusiones mucho mayores en el atajo del problema.

En nuestra muestra aparece en mayor porcentaje el perfil de defensa de la víctima que el de complicidad. Desafortunadamente, los espectadores se comportan más a menudo como cómplices debido a las recompensas sociales o a las aprobaciones que reciben por parte de las personas agresoras y que suelen gozar de un cierto estatus social (Salmivalli, Kärnä y Poskiparta 2011).

\section{Aspectos innovadores para la acción educativa}

Los resultados obtenidos alientan la necesidad de emprender acciones educativas que ayuden a paliar el fenómeno de la ciberviolencia de género.

Una de las acciones a considerar se centra en la formación del profesorado. Estudios empíricos (Ahtola, Haataja, Kärnä, Poskiparta y Salmivalli, 2012), han demostrado que el profesorado tiene un conocimiento incompleto sobre el ciberacoso, en ocasiones inexacto y que a menudo carece de habilidades para intervenir. Por ejemplo, el profesorado no siempre reconoce las situaciones críticas del ciberacoso, dado que este se produce en el entorno virtual y por medio de una tecnología. Sin embargo, los profesores son un elemento principal para llevar a cabo estos cambios en el entorno educativo. El profesorado es importante por varias razones. Primera, los efectos que puede tener sobre el alumnado pueden expandirse en el tiempo, pues chicos y chicas permanecen años en el centro escolar y es en éste donde debe comenzar la prevención para evitar el aumento del acoso a medida que aumente la edad. Segunda, el conocimiento de los maestros sobre la intimidación y su compromiso para abordarlo son esenciales para que un programa de intervención tenga éxito. Tercera, el profesorado puede realizar al mismo tiempo una labor con las familias. Por todo ello, consideramos que la formación de los agentes educativos es primordial.

Otra de las acciones necesarias es la elaboración de materiales y guías para ayudar tanto a agentes educativos como sociales. Las guías deberían incluir datos de la incidencia del ciberacoso, los tipos, la frecuencia de comportamientos, la relación entre offline y online, los perfiles cibernéticos de agresores, las estrategias de intervención que pueden apoyar a las víctimas y/o reducir el ciberacoso y los mecanismos de afrontamiento necesarios.

Por último, queremos enfatizar la necesidad de que las intervenciones que se realicen no deben estar centradas en los dos polos del fenómeno del ciberacoso, agresor y víctima, sino incluir a todas las personas participantes. Como hemos visto en la literatura los comportamientos y actitudes de las personas espectadoras son muy importantes a la hora de paliar en parte el impacto sobre la víctima, un impacto que se expande a todo el entorno, lo cual es de una gran importancia en los centros educativos. 


\section{REFERENCIAS BIBLIOGRÁFICAS}

Ahtola, A., Haataja, A., Kärnä, A., Poskiparta, E. y Salmivalli, C. (2012). For children only? Effects of the KiVa antibullying program on teachers. Teaching and Teacher Education, 28, 851859. DOI: https://doi.org/10.1016/j.tate.2012.03.006

Arseneault, L., Bowes, L. y Shakoor, S. (2010). Bullying Victimization in Youths and Mental Health Problems: "Much Ado About Nothing"? Psychological, Medicine, 40(5), 717-729. DOI: https://doi.org/10.1017/S0033291709991383

Berlanga, V., Rubio, M. J. y Vilà, R. (2013). Cómo aplicar árboles de decisión en SPSS. REIRE -Revista educativa d'Innovació i Recerca en Educació, 6(1), 65-79.

Calmaestra, J. (2011). Cyberbullying: prevalencia y características de un nuevo tipo de bullying indirecto. Tesis doctoral. Servicio de Publicaciones de la Universidad de Córdoba.

Calvete, E., Orue, I., Estévez, A., Villardón, L. y Padilla, P. (2010). Cyberbullying in adolescents: Modalities and aggressors' profile. Computers in Human Behavior, 26, 1128-1135. DOI: https://doi.org/10.1016/j.chb.2010.03.017

Cooper, K., Quayle, E., Jonsson, L., y Svedin, C. G. (2016). Adolescents and self-taken sexual images: A review of the literature. Computers in Human Behavior, 55, 706-716. DOI: https://doi. org/10.1016/j.chb.2015.10.003

Donoso, T. (Coord.) (2014). Violencias de género 2.0. Barcelona: Kit-book. http://gredidona.blogspot.com.es/p/publicacions.html

Donoso, T., Vilà, R., Rubio, M. J. y Prado, N. (2016). Perfil de cibervictimización ante las violencias de género 2.0 Femeris, 1(2), 35-57. DOI: https://doi.org/10.20318/femeris.2016.3226

Ersilia, M., Modena, M. y Tani, F. (2009). Bullying and Victimization in Adolescence: Concurrent and Stable Roles and Psychological Health Symptoms. Journal of Genetic Psychology, 170(2), 115-133. DOI: https://doi.org/10.3200/GNTP.170.2.115-134

Estébanez, I. y Vázquez, N. (2013). La desigualdad de género y el sexismo en las redes sociales. Administración de la Comunidad Autónoma del País Vasco. Departamento de Educación, Política Lingüística y Cultura.

Finn, J. y Atkinson, T. (2009). Promoting the safe and strategic use of technology for victims of intimate partner violence: Evaluation of the Technology Safety Project. Violence againts women, 15(11), 1402-1414. DOI: https://doi.org/10.1177/1077801209346723

Garmendia, M., Garitaonandia, C., Martínez, G. y Casado, M. A. (2011). EU Kids Online II: Mejorando el conocimiento sobre el uso y la seguridad en internet de los menores en Europa. Disponible en: www.ehu.es/eukidsonline

Gianluca, G., Pozzoli, T., Borghi, F. y Franzoni, L. (2008). The Role of Bystanders in Students' Perception of Bullying and Sense of Safety. Journal of School Psychology, 46(6), 617-638. DOI: https://doi.org/10.1016/j.jsp.2008.02.001

Gini, G., Pozzoli,T., Borghi, F. y Franzoni, L. (2008). The Role of Bystanders in Students' Perception of Bullying and Sense of Safety. Journal of School Psychology, 46(6), 617-638. DOI: https://doi.org/10.1016/i.jsp.2008.02.001

Hand, T., Chung, D. y Peters, M. (2009). The Use of Information and Communication Technologies to Coerce and Control in Domestic Violence and Following Separation. Disponible en: https://advocacy4oromia.files.wordpress.com/2015/03/stakeholderpaper 6.pdf 
Instituto Nacional de Estadística (2015). Notas de Prensa: Encuesta sobre Equipamiento y Uso de Tecnologías de Información y Comunicación en los Hogares. Madrid:INE.

Instituto Nacional de Estadística (2016). Notas de Prensa: Encuesta sobre Equipamiento y Uso de Tecnologías de Información y Comunicación en los Hogares. Madrid:INE.

Martínez, E., Sendín, J. C. y García, A. (2013). Percepción de los riesgos en la red por los adolescentes en España: usos problemáticos y formas de control. Anàlisi Monogràfic, 111-130.

Méndez, M. J., Villar, M. y Barreiro, F. (2015). Estudio de los espacios virtuales como soportes para la violencia de género en la adolescència. Revista de Estudios e Investigación en Psicología y Educación, Extr (13), 172-175. DOI: https://doi.org/10.17979/reipe.2015.0.13.525

Menesini, E., Nocentini, A. y Palladino, B. (2012). Empowering Students Against Bullying and Cyberbullying: Evaluation of an Italian Peer-led Model. International Journal of Conflict and Violence, 6(2), $314-321$.

Ortega, R., Calmaestra, J. y Mora, J. A. (2008). Cyberbullying. International Journal of Psychology and Psychological Therapy, 8(2), 183-192.

Pérez López, C. (2011). Técnicas de segmentación. Conceptos, herramientas y aplicaciones. Madrid: Gaceta Grupo Editorial.

Pérez López, C. y Santín, D. (2007). Minería de Datos: Técnicas y Herramientas. Madrid: Ediciones Paraninfo.

Román, M. V. y Lévy, J. P. (2003). Clasificación y segmentación jerárquica. En Lévy, J. P. y Varela, J. (Eds). Análisis multivariable para las Ciencias Sociales (pp.567-630). Madrid: Pearson Educación.

Salmivalli, C., Kärnä, A. y Poskiparta, E. (2011). Counteracting bullying in Finland: The KiVa program and its effects on diferent forms of being bullied. International Journal of Behavioral Development. 35(5) 405-411. DOI: https://doi.org/10.1177/0165025411407457

Salmivalli, C. y Poskiparta, E. (2012). Making bullying prevention a priority in Finnish schools: The KiVa antibullying program. New Directions for Student Leadership, 133, 41-53, DOI: https:// doi.org/10.1002/yd.20006

Smith, P. K. (2006). CIBERACOSO: naturaleza y extensión de un nuevo tipo de acoso dentro y fuera de la escuela. Congreso de Educación de Palma de Mallorca. Palma de Mallorca.

Ttofi, M. M., Farrington, D.P., Lösel, F. y Loeber, R. (2011). The Predictive Efficiency of School Bullying versus Later Offending: A Systematic/ Meta-Analytic Review of Longitudinal Studies. Criminal Behavior and Mental Health, 21(2), 80-89. DOI: https://doi.org/10.1002/cbm.808

Veenstra, R., Siegwart, L., Munniksma, A. y Dijkstra, J. K. (2010). The Complex Relation between Bullying, Victimization, Acceptance, and Rejection: Giving Special Attention to Status, Affection, and Sex Differences. Child Development , 81, 480-86. DOI: https://doi. org/10.1111/j.1467-8624.2009.01411.x

White, D. S. y Le Cornu, A. (2011). Visitors and Residents: A new typology for online engagement. First Monday, 16(9). DOI: https://doi.org/10.5210/fm.v16i9.3171 